\title{
Erratum: Diphoton Production at Hadron Colliders: A Fully Differential QCD Calculation at Next-to-Next-to-Leading Order [Phys. Rev. Lett. 108, 072001 (2012)]
}

\author{
Stefano Catani, Leandro Cieri, Daniel de Florian, Giancarlo Ferrera, and Massimiliano Grazzini \\ (Received 5 July 2016; published 16 August 2016)
}

DOI: 10.1103/PhysRevLett.117.089901

The next-to-next-to-leading-order (NNLO) results presented in Table I and Figs. 1-3 of the Letter are affected by an error in the numerical code that implements the NNLO calculation. The corrected results are reported below. Accompanying quantitative statements in the text have to be modified accordingly, as follows: two lines above Fig. 1, "of about $K \simeq 1.35$. We find that about $30 \%$ of the NNLO corrections is due to the $g g$ channel (the box contribution is responsible for more than half of it)" should be replaced by "of about $K \simeq 1.25$. We find that about $13 \%$ of the NNLO corrections is due to the total contribution of the $g g$ channel (which is roughly two thirds of the box contribution)" at the end of the second-to-last paragraph, "roughly 30\%" should be replaced by "roughly 20\%"; and in the middle of the last paragraph, "of about $30 \%-40 \%$ " should be replaced by "of about 20\%-30\%".

TABLE I. Cross sections for $p p \rightarrow \gamma \gamma+X$ at the LHC $(\sqrt{s}=14 \mathrm{TeV})$, including uncertainties of the numerical calculation. The applied cuts are described in the text.

\begin{tabular}{lccr}
\hline \hline$\sigma(\mathrm{fb})$ & LO & NLO & NNLO \\
\hline$\mu_{F}=\mu_{R}=M_{\gamma \gamma} / 2$ & $5045 \pm 1$ & $26581 \pm 23$ & $42238 \pm 330$ \\
$\mu_{F}=\mu_{R}=M_{\gamma \gamma}$ & $5712 \pm 2$ & $26402 \pm 25$ & $40269 \pm 250$ \\
$\mu_{F}=\mu_{R}=2 M_{\gamma \gamma}$ & $6319 \pm 2$ & $26045 \pm 24$ & $38901 \pm 310$ \\
\hline \hline
\end{tabular}

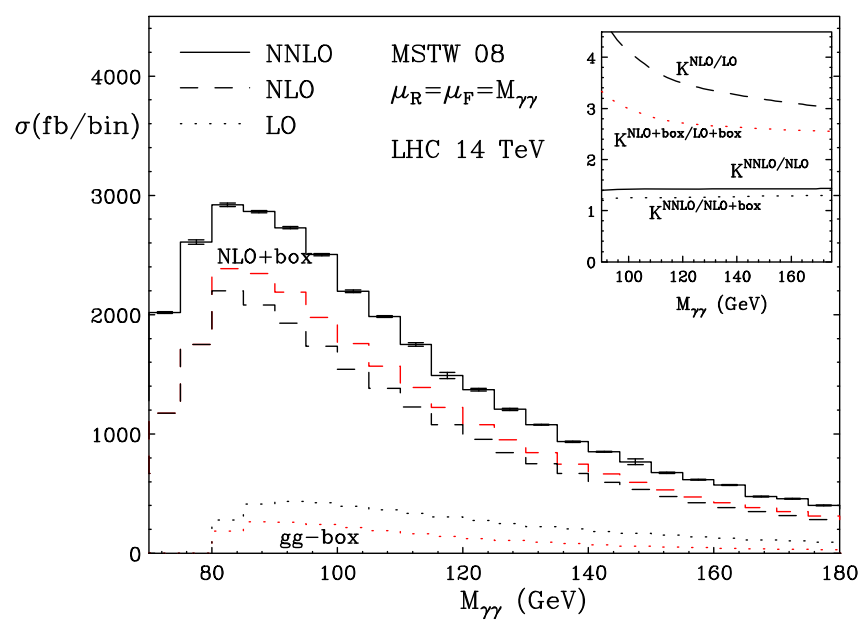

FIG. 1. Invariant mass distribution of the photon pair at the LHC $(\sqrt{s}=14 \mathrm{TeV}$ ): LO (dots), NLO (dashes), and NNLO (solid) results with corresponding statistical uncertainties of the Monte Carlo integration. We also present the results of the box and NLO + box contributions. The inset plot shows the corresponding K-factors. 


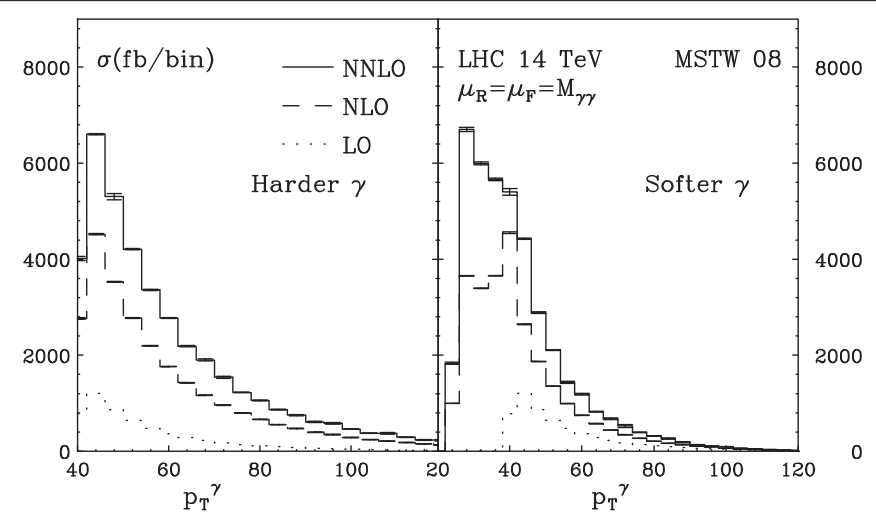

FIG. 2. Transverse-momentum distribution of the harder (left) and softer (right) photon at the LHC (notation as in Fig. 1).

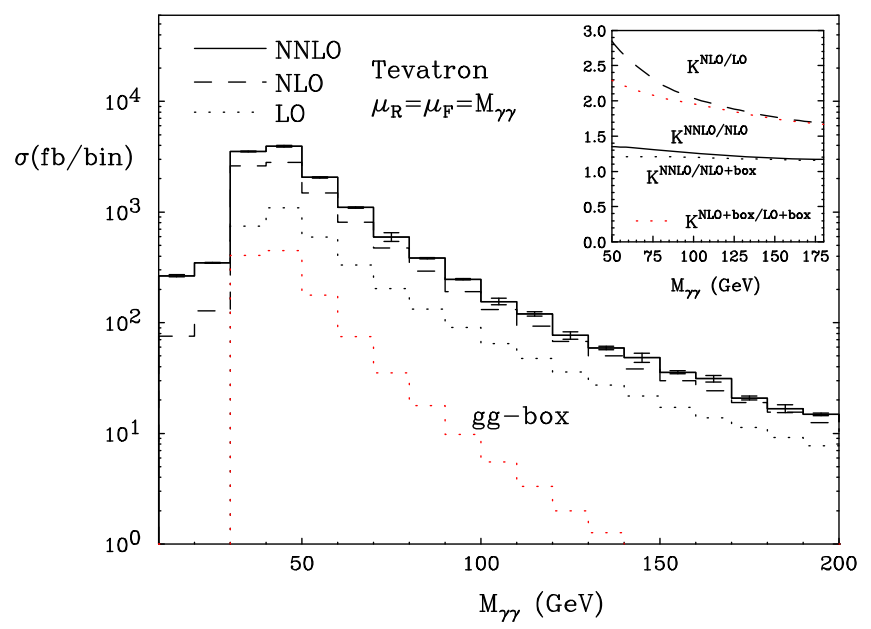

FIG. 3. Same as Fig. 1, but for Tevatron kinematics (as described in the text). 